\title{
Valgus Osteotomy with DHS Fixation in the Management of Malunited Intertrochanteric Fractures in a Rural Population
}

\author{
Subash Y, DNB \\ Department of Orthopaedics, Saveetha University Saveetha Medical College and Hospital, Kanchipuram, India
}

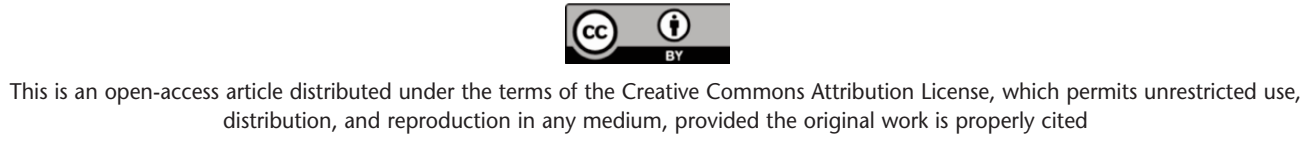

Date of submission: 02nd August 2019

Date of acceptance: 18th September 2020

\begin{abstract}
Introduction: Malunited intertrochanteric fractures are frequently seen in rural populations as they tend to go in for native treatment with traditional bone setters. The resulting Coxa vara is associated with shortening, abductor weakness, limp and decreased range of movement of the affected hip. The aim of this study was to evaluate the role of Valgus osteotomy with Dynamic hip screw (DHS) fixation in the management of these fractures and to evaluate the functional outcome using the Harris hip score.

Materials and Methods: Fifteen patients with malunited intertrochanteric fractures who presented between January 2011 to January 2013 were managed by Valgus osteotomy with DHS fixation and were followed-up for a minimum period of three years.

Results: There was a male preponderance seen in our study with the right hip being more commonly affected. The most common mode of injury was slip and fall followed by road traffic accidents. The duration of native treatment ranged from seven to 12 weeks and the time of presentation to the hospital ranged from four to nine months following injury. Pre-operative mean neck shaft angle was $94.73^{\circ}$ while it was $134.6^{\circ}$ post-operatively. The mean pre-operative Harris hip score was 72.33 and it was 91 at follow-up. All patients were happy with the procedure and the functional outcome.

Conclusion: Valgus osteotomy with DHS fixation is an effective procedure in the management of malunited intertrochanteric fractures. It corrects the limb length discrepancy, restores the decreased neck shaft angle, improves range of movement, restores the integrity of the abductor mechanism of the hip and gives good functional results.
\end{abstract}

Keywords:

intertrochanteric fractures, malunion, valgus osteotomy, DHS

\section{INTRODUCTION}

Intertrochanteric fractures of the hip are common fractures seen in an elderly age group due to poor bone stock while in younger individuals they often result from high velocity trauma such as fall from a height and road traffic accidents ${ }^{1,2}$. Various surgical options are available in the management of these fractures. In developing countries patients do tend to present late for treatment due to various factors. Our hospital is located in a rural area where most of the population belong to a lower socio economic group and a bulk of them are manual laborers who depend on daily wages for their sustenance. So, factors such as lack of affordability and also traditional belief in native bone setters make them go in for native treatment with crude native splints extending from the groin to the toes. The period of native treatment often lasts for around six to eight weeks with the splints being changed at intervals of two weeks. There is often a history of massage or manipulation before the splints are applied leading to an increased risk of development of myositis ossificans. Since the intertrochanteric region is composed primarily of cancellous bone these fractures tend to unite well albeit in a nonanatomic manner leading to a malunion. The resulting Coxa vara is associated with problems such as shortening, external rotation deformity with limitation of abduction and internal rotation, limp, and issues such as hip and back pain due to mechanical factors ${ }^{3-5}$.

Due to decrease in the range of movement these patients find it difficult to squat and sit cross legged which are necessary for them for purposes of defecation and for sitting on the ground for partaking meals respectively and hence it becomes a disability for them which brings them to the hospital for treatment at a later stage.

The ideal treatment for failed and malunited intertrochanteric fractures as mentioned in literature has been a total hip

Corresponding Author: Yeshwanth Subash, Department of Orthopaedics Surgery, Saveetha University Saveetha Medical College and Hospital, Kuthambakkam, Tamil Nadu 600124, India

Email: djyesh@rediffmail.com 
arthroplasty but it would not be ideal in this situation because it is an expensive procedure and the lack of affordability and the avoidance of squatting and sitting cross legged following arthroplasty which would not be feasible for the patients who present from a rural population. A review of literature as well as articles from various authors have not described any other procedure in the management of these malunited fractures. Hence there is a need for a procedure which will restore limb length discrepancy, bring back the reduced neck shaft angle to as near normal as possible, improve the range of movement of the hip facilitating squatting and sitting cross legged and restore the integrity of the abductor mechanism and which gives good reproducible results and is also cost effective to the patient. In this scenario, Valgus osteotomy with DHS fixation would be ideally suited and would address all the above mentioned issues.

Valgus osteotomy is an established procedure which has been primarily used in the management of femoral neck fractures especially in the younger population where there arises a need to salvage the femoral head. It has not been described traditionally in the management of these malunited intertrochanteric fractures. The aim of this study was to evaluate the role of this procedure in the management of malunited intertrochanteric fractures and to analyse the functional results using the Harris hip score.

\section{MATERIALS AND METHODS}

This was a prospective study of 15 patients with malunited intertrochanteric fractures managed by Valgus osteotomy with DHS fixation between January 2011 to January 2013. This study was approved by the ethical committee of our institution All patients with malunited intertrochanteric fractures who were willing for the procedure and for regular follow-up were included in the study while patients not willing for follow-up and patients with active hip or systemic infection were excluded. On presentation to the hospital a thorough physical examination was done and findings such as amount of shortening, range of motion of the affected hip and the pre-operative Harris hip score were assessed and documented in the case records. The patients were then evaluated radiologically by taking radiographs of the pelvis with both hips anteroposterior view and affected hip lateral view and the neck shaft angle was measured and documented. Extensive pre-operative planning was not done because the aim was not to take a wedge of bone but only to perform a sliding osteomy at the subtrochanteric level in order to minimise shortening. All patients were then worked up for the surgical procedure.

The patients were then taken up for surgery after obtaining anaesthetic fitness and proper informed consent for the procedure. The surgery was performed under spinal anaesthesia with the patient in the supine position on the fracture table under fluoroscopic guidance. The proximal femur was exposed via a standard lateral approach and after making an entry point using a $3.2 \mathrm{~mm}$ drill bit, a guide wire was passed in the centre of the femoral neck and position was confirmed in anteroposterior and lateral views (Fig. 1 and 2). Reaming was then performed with a triple reamer and an appropriate size Richards screw was then applied. No effort was made to osteotomize the fracture site. An oblique osteotomy was then performed using an oscillating saw at a level just distal to the level of the lesser trochanter without taking a wedge of bone in order to minimise post-operative shortening (Fig. 3 and 4). Care was taken not to perform the osteotomy too distal to the subtrochanteric region in order to avoid problems with union at the osteotomy site. After performing the osteotomy, the external rotation deformity was corrected, a 5 holed DHS plate with a short barrel was then applied, the limb was taken into abduction and traction was reduced in order to reduce the shaft to the proximal fragment and the plate was fixed with five $4.5 \mathrm{~mm}$ cortical screws. A DHS with a standard $135^{\circ}$ angle was used in all cases. The limb was then brought back to a neutral position where the restoration of the limb length as well as the neck shaft angle could be appreciated. After ensuring heamostasis and placing a drain insitu the wound was closed in layers and sterile dressing was applied.

The patients were made to sit up in bed the same evening of surgery and the knee and ankle were actively mobilised. They were made to stand and walk on the first or second post-operative day with non-weight bearing walking with walking frame support subjective to pain tolerance and patient compliance. Drain removal was done on the third day and intravenous antibiotics were given for three days postoperatively. Wound inspections were done on the third and sixth post-operative day followed by suture removal on the 10th post-operative day. Limb length measurement, range of movement of the hip and the neck shaft angle on the postoperative radiographs were noted and documented. Patients were then started on quadriceps and hip abductor strengthening exercises and on discharge were asked to continue strict non-weight bearing until advised and were asked to review for follow-up at one, three, six months and at yearly intervals for three years. Radiological evaluations were done by taking serial radiographs at follow-up and functional evaluation was done using the Harris hip score and documented. Trendelenberg's test was performed in all patients to assess the integrity of the abductor mechanism. The data collected was analysed using IBM [SPSS Version 22.0. Armonk, NY: IBM Corp]. Chi square test was used in the comparison of categorical variables. A P value of less than 0.05 was considered to be statistically significant.

\section{RESULTS}

There were 15 patients in our study with 13 males and two females. The right hip was more commonly affected as seen in nine patients. The age of the patients ranged from 49 to 70 
Table I: Patient demographics and data

\begin{tabular}{|c|c|c|c|c|c|c|c|c|c|}
\hline \multirow[b]{2}{*}{$\begin{array}{l}\text { Serial } \\
\text { number }\end{array}$} & \multirow[b]{2}{*}{$\begin{array}{l}\text { Agel } \\
\text { sex }\end{array}$} & \multirow[b]{2}{*}{$\begin{array}{l}\text { Mode of } \\
\text { injury }\end{array}$} & \multicolumn{2}{|c|}{$\begin{array}{l}\text { Neck shaft angle } \\
\text { (degrees) }\end{array}$} & \multicolumn{2}{|c|}{$\begin{array}{l}\text { Shortening } \\
\text { (cm) }\end{array}$} & \multicolumn{2}{|c|}{ Harris hip score } & \multirow{2}{*}{$\begin{array}{c}\text { Osteotomy } \\
\text { site } \\
\text { union (weeks) }\end{array}$} \\
\hline & & & Pre-op & Post-op & Pre-op & Post-op & Pre-op & Post-op & \\
\hline 1 & $54 / \mathrm{M}$ & RTA & 90 & 135 & 3 & 0.5 & 48 & 76 & 13 \\
\hline 2 & $56 / \mathrm{M}$ & RTA & 91 & 134 & 3 & 0.4 & 47 & 79 & 11 \\
\hline 3 & $49 / F$ & $\mathrm{FFH}$ & 95 & 135 & 2.7 & 0 & 50 & 84 & 11 \\
\hline 4 & $64 / \mathrm{M}$ & SAF & 100 & 140 & 2 & 0 & 51 & 79 & 12 \\
\hline 5 & $70 / \mathrm{M}$ & SAF & 102 & 136 & 2.1 & 0 & 47 & 82 & 13 \\
\hline 6 & $65 / \mathrm{M}$ & SAF & 98 & 134 & 2.8 & 0 & 48 & 84 & 13 \\
\hline 7 & $59 / F$ & RTA & 95 & 135 & 3 & 0.2 & 55 & 77 & 15 \\
\hline 8 & $62 / M$ & SAF & 96 & 134 & 2.6 & 0 & 49 & 81 & 14 \\
\hline 9 & $64 / \mathrm{M}$ & SAF & 92 & 134 & 2.6 & 0 & 48 & 79 & 11 \\
\hline 10 & $70 / \mathrm{M}$ & SAF & 90 & 135 & 3.1 & 0 & 53 & 75 & 12 \\
\hline 11 & $63 / \mathrm{M}$ & RTA & 98 & 137 & 2.8 & 0 & 49 & 80 & 15 \\
\hline 12 & $59 / \mathrm{M}$ & FFH & 94 & 134 & 2.5 & 0 & 50 & 79 & 15 \\
\hline 13 & $61 / \mathrm{M}$ & SAF & 96 & 135 & 2.7 & 0 & 47 & 84 & 11 \\
\hline 14 & $69 / \mathrm{M}$ & SAF & 90 & 135 & 3 & 0 & 54 & 83 & 12 \\
\hline 15 & $69 / \mathrm{M}$ & RTA & 94 & 130 & 2 & 0 & 50 & 80 & 13 \\
\hline
\end{tabular}

*RTA-Road traffic accident. SAF-Slip and fall. FFH-Fall from a height

Table II: The pre and post-operative range of movement

\begin{tabular}{|lcccccccccc|}
\hline S.No & \multicolumn{4}{c}{ Pre-operative range of movement } & \multicolumn{5}{c|}{ Post-operative range of movement } \\
& Flex & Abd & Add & IR & ER & Flex & Abd & Add & IR & ER \\
\hline 1 & 90 & 12 & 15 & 5 & 10 & 120 & 40 & 25 & 40 & 25 \\
2 & 85 & 10 & 20 & 6 & 15 & 110 & 42 & 20 & 42 & 30 \\
3 & 87 & 14 & 20 & 10 & 20 & 100 & 45 & 21 & 39 & 35 \\
4 & 80 & 9 & 25 & 11 & 15 & 120 & 39 & 20 & 44 & 45 \\
5 & 75 & 11 & 20 & 8 & 14 & 110 & 40 & 25 & 40 & 40 \\
6 & 70 & 10 & 15 & 6 & 21 & 100 & 38 & 20 & 41 & 35 \\
7 & 86 & 12 & 21 & 9 & 20 & 120 & 41 & 20 & 42 & 40 \\
8 & 70 & 13 & 15 & 10 & 15 & 110 & 42 & 24 & 44 & 41 \\
9 & 85 & 10 & 25 & 10 & 18 & 100 & 37 & 21 & 41 & 45 \\
10 & 80 & 9 & 20 & 12 & 21 & 110 & 40 & 20 & 43 & 40 \\
11 & 90 & 15 & 15 & 11 & 15 & 120 & 42 & 20 & 40 & 38 \\
12 & 80 & 11 & 20 & 13 & 20 & 100 & 45 & 25 & 39 & 40 \\
13 & 85 & 9 & 15 & 9 & 21 & 110 & 44 & 21 & 38 & 45 \\
14 & 75 & 10 & 18 & 12 & 20 & 100 & 40 & 25 & 42 & 42 \\
15 & 70 & 12 & 24 & 11 & 18 & 100 & 42 & 20 & 45 & 45 \\
\hline
\end{tabular}

* Flex-Flexion. Abd-Abduction. Add-Adduction. IR-Internal rotation. ER-External rotation

years with the mean age being 61.53 years. The most common mode of injury was slip and fall followed by road traffic accidents and fall from a height. The time from injury to presentation to the hospital ranged from four to nine months with the mean being 6.2 months. The duration of native treatment ranged from seven to 12 weeks with the mean being 9.33 weeks. The average neck shaft angle preoperatively was $94.73^{\circ}$ ranging from $90^{\circ}$ to $102^{\circ}$ while it was $134.6^{\circ}$ in the post-operative period ranging from $130^{\circ}$ to $140^{\circ}$. The average pre-operative shortening was $2.66 \mathrm{~cm}$ ranging from two to $3.1 \mathrm{~cm}$ while three patients had minimal shortening in the post-operative period of about 0.2 to $0.5 \mathrm{~cm}$ which was well tolerated by the patients. The average preoperative Harris hip score was 72.33 ranging from 61 to 80 while it was 91 in the post-operative period ranging from 80 to 97 with a gain of 18.67 points. The average duration of surgery was 98.5 minutes and the average blood loss was 209.4 milliliters. The average time to union of the osteotomy site was 12.7 weeks ranging from 11 to 15 weeks (Table I). All patients had limitation of abduction and internal rotation pre-operatively which improved significantly in the postoperative period with the mean flexion of more than $90^{\circ}$, abduction $-40^{\circ}$, adduction- $25^{\circ}$, internal rotation $-45^{\circ}$, external rotation- $30^{\circ}$ (Fig. 5,6 and 7 and Table II). There were no complications such as superficial or deep infection, screw backout, joint penetration, loss of fixation or correction or non-union at the osteotomy site encountered in our study. None of the patients were lost to follow-up. All patients were happy with the procedure and the functional outcome. 


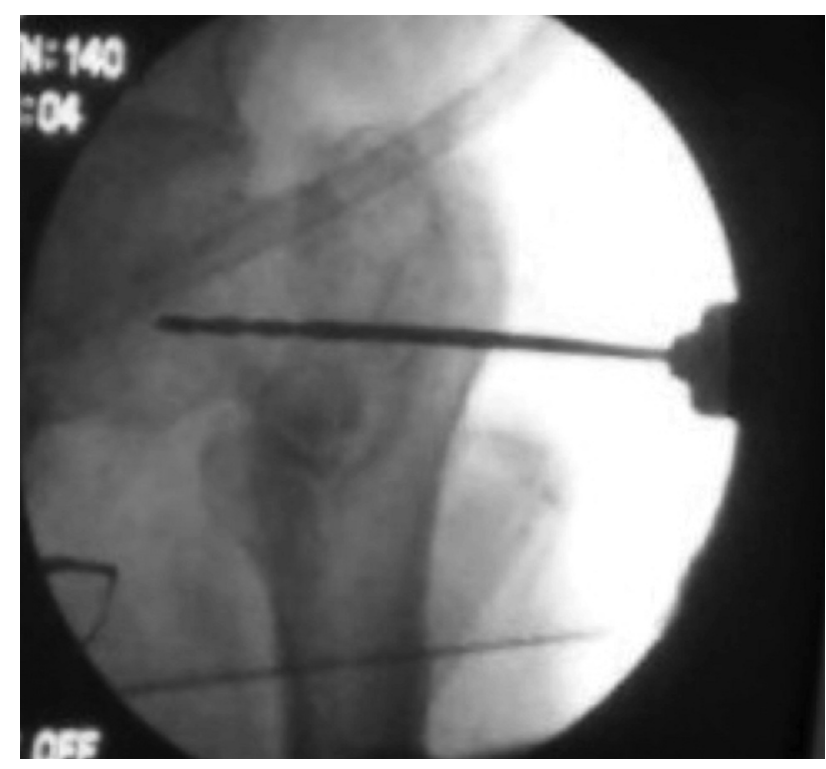

Fig. 1: Entry point made using a $3.2 \mathrm{~mm}$ drill bit.

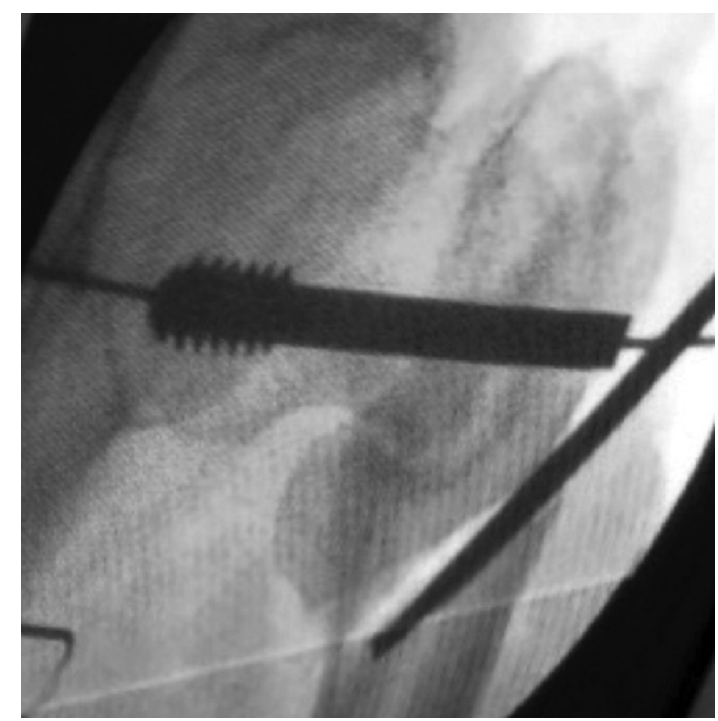

Fig. 3: Richards screw inserted and osteotomy performed at the subtrochanteric level.

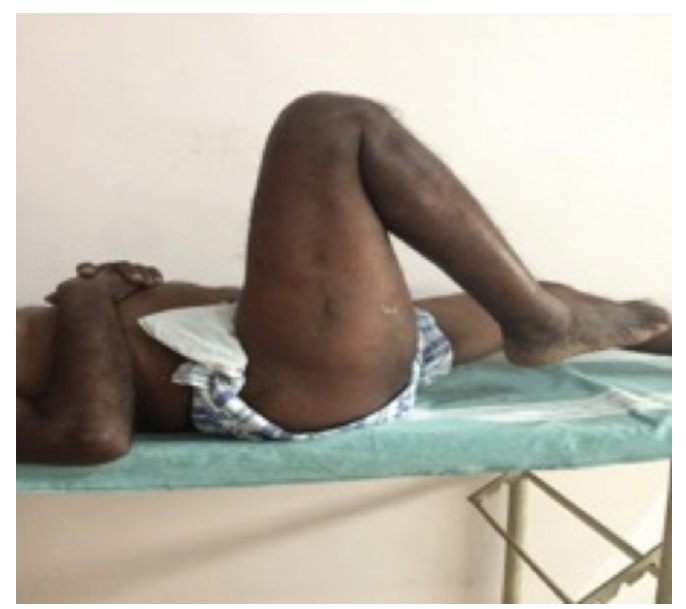

Fig. 5: Range of motion-Hip flexion.

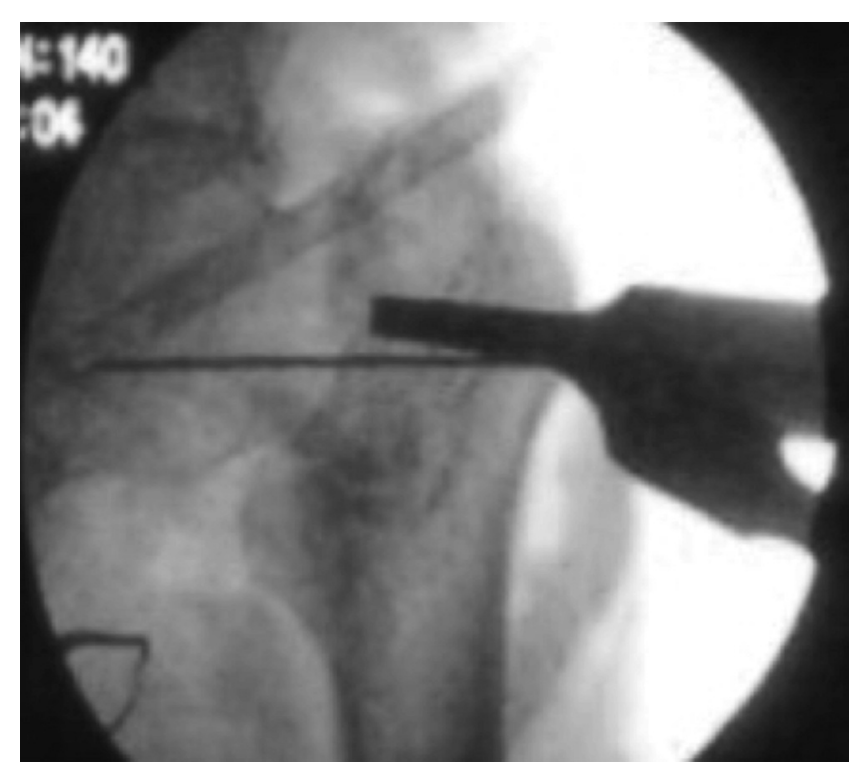

Fig. 2: Guide wire passed parallel and inferior to the femoral neck under fluoroscopic guidance.

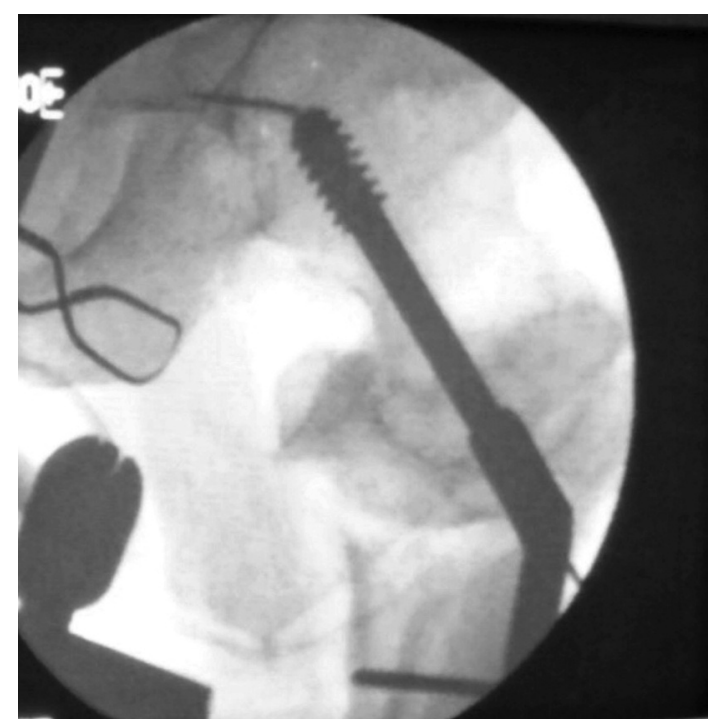

Fig. 4: DHS barrel and plate applied with the restoration of the neck shaft angle to an anatomic position.

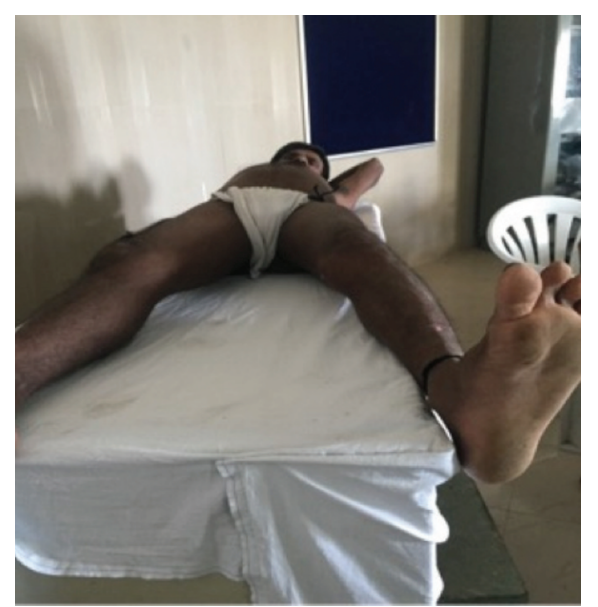

Fig. 6: Range of motion-Hip Abduction. 


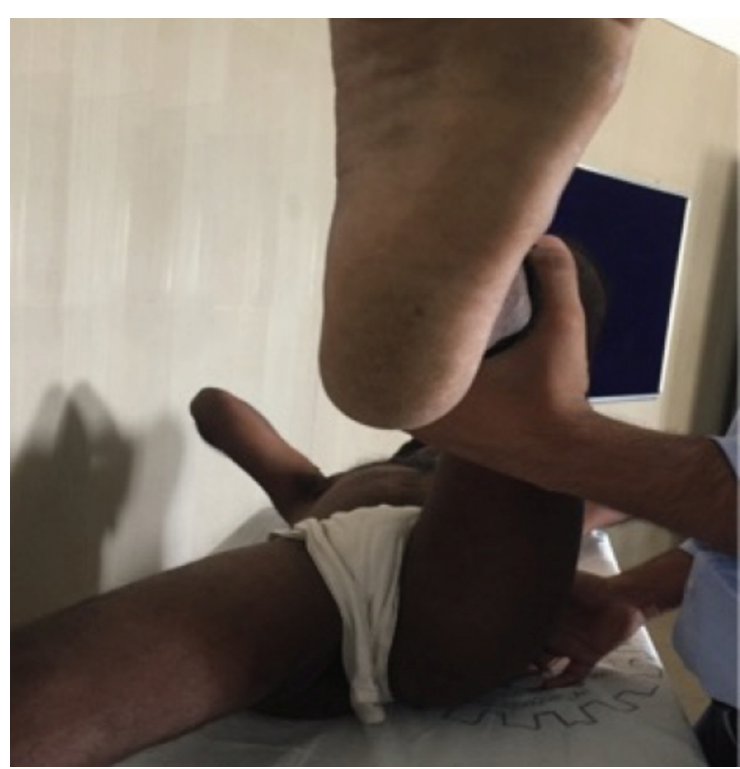

Fig. 7: Range of motion-Hip Internal rotation.

\section{DISCUSSION}

The management of fresh intertrochanteric fractures are quite straight forward with various treatment options and implants available for the same. In case of failed management of the fractures, the patients present with a malunion with coxa vara which results in mechanical block with restriction of abduction and internal rotation as well as shortening of the affected limb which results in weakness of the abductor mechanism of the affected hip. Total hip arthroplasty has been described as the ideal procedure in the management of these fractures and has been remains the gold standard in its treatment ${ }^{6}$. The other option to be considered in the management of these fractures would be to perform an osteotomy at the fracture site to undo the malunion and proceed to fix it with either surface or intramedullary implants but it would require more extensive dissection and would be associated with extensive bleeding as well as longer surgical time. Other procedures for the management of malunited fractures have not been described in a review of literature. In this scenario management of malunited intertrochanteric fractures posts a challenge regarding treatment options. Even though these patients don't have significant pain, they have issues such as shortening of the affected limb, trendelenbergs gait due to proximal migration of the greater trochanter and abductor weakness and mechanical factors such as decrease of range of motion of the affected hip resulting in difficulty or inability to squat or sit cross legged which is disabling to the patient. All 15 patients in our study belonged to a rural population who are primarily involved in agricultural work and often depend on daily wages to support themselves.

The ability to squat and sit cross legged is essential for them to carry out their activities of work as well as daily living to the best possible extent. Hence total hip arthroplasty would not be an ideal treatment option for them due to factors such as lack of affordability and avoidance of squatting and sitting cross legged following the procedure which is an essential requisite for the study population. Valgus osteotomy is an established procedure which is usually performed in femoral neck non-unions and has not traditionally described for malunited intertrochanteric fractures. This osteotomy is performed at the subtrochanteric level and no effort is made to open the fracture site to undo the malunion. Valgus osteotomy has the advantages of correcting the limb length discrepancy, restores the reduced neck shaft angle to near normal, improves the range of movement and gives good functional results bringing about an improvement in the quality of the life for the patient enabling them to carry out their activities of work as well as daily living to the best possible extent without any restriction. In our procedure, we did not take a wedge of bone as described in the classical form of the procedure but instead we performed a sliding osteotomy in order to maintain length and to avoid shortening. We emphasise the fact that a sliding osteotomy is superior to a wedge osteotomy as it helps in maintain limb length to the best possible extent and also the fixation with a DHS is quite easy and straightforward as compared to an angle blade plate which is technically demanding and has quite a steep learning curve ${ }^{7-10}$. Fixation following osteotomy can be done with either a DHS or an angled blade plate. We routinely used a DHS with a fixed $135^{\circ}$ angle as we found it to be technically easier to perform as compared to a blade plate which is more technically demanding.

In our study, there was a good restoration of the neck shaft angle to a near normal level in all patients and also good correction of the limb length discrepancy was achieved. Mild shortening was seen in three of our patients but it was quite well tolerated by them without any issues. There was a good improvement in the gait pattern in all patients following union of the osteotomy site and starting of full weight bearing walking which was at an average of 12.7 weeks. All patients had a significant improvement in the range of movement of the affected hip in the post-operative period $(\mathrm{P}<0.005)$ and at the end of three months they were able to squat and sit cross legged quite comfortably which was quite satisfactory for them. The increase in the range of motion of the affected hip is due to the better arc of movement and also due to the correction of the mechanical block of abduction and internal rotation caused by the coxa vara resulting from the malunion of these fractures. There was also an improvement in the Harris hip score with a gain of 18.67 points in the post-operative period. There were no iatrogenic complications seen in our study and none of our patients were lost to follow-up. All patients were followed-up for a three years period and were doing well functionally at the end of the last follow-up. A review of literature showed that valgus osteotomy with DHS fixation was performed mostly for non-unions of the femoral neck with adequate literature supporting the same $\mathrm{e}^{11-13}$. 
The limitations of our study were a small study sample with a relatively short follow-up period. A larger study group would ideally be essential to observe whether the beneficial effects of the osteotomy are uniform and consistent. Through this study, we conclude that Valgus osteotomy with DHS fixation is an effective treatment option in the management of malunited intertrochanteric fractures of the femur. It corrects the limb length discrepancy, restores the neck shaft angle and improves the range of motion thereby improves the function of the affected hip and improves the quality of life for the patient. It can be used as a good and reliable alternative in patients for whom Total hip arthroplasty would not be indicated.

\section{CONCLUSION}

Valgus osteotomy with DHS fixation is an effective procedure in the management of malunited intertrochanteric fractures. It corrects the limb length discrepancy, restores the decreased neck shaft angle, improves range of movement, restores the integrity of the abductor mechanism of the hip and gives good functional results.

\section{CONFLICT OF INTEREST}

The authors declare no potential conflicts of interest.

\section{REFERENCES}

1. Filipov O. Epidemiology and social burden of the femoral neck fractures. J of IMAB. 2014; 20(4): 516-8. doi: 10.5272/jimab.2014204.516

2. Massie WK. Fracture of the hip. J Bone Joint Surg Am. 1964; 46: 658-90

3. Lifeso R, Younge D. The neglected hip fracture. J Orthop Trauma. 1990; 4: 287-92. doi: 10.1097/00005131-199004030-00009

4. Barnes B, Dunovan K. Functional outcomes after hip fracture. Phys Ther. 1987; 67(11): 1675-9. doi: 10.1093/ptj/67.11.1675

5. Johnston JD, Noble PC, Hurwitz DE, Andriacchi TP. Biomechanics of the hip. In: Callaghan J, Rosenberg AG, Rubas HE, Editors. The Adult Hip. Philidalphia: Lippincott Williams \& Wilkins; 1998. p. 81-90.

6. Haidukewych GJ, Berry DJ. Hip arthroplasty for salvage of failed treatment of intertrochanteric hip fractures. J Bone Joint Surg Am. 2003; 85(5): 899-904. doi: 10.2106/00004623-200305000-00019

7. Muller ME. Intertrochanteric osteotomy. Indication, preoperative planning, technique. In: Schatzker J, editor. The intertrochanteric osteotomy. Berlin: Springer-Verlag Berlin Heidelberg; 1984. p. 25-66.

8. Bartonicek J, Skala-Rosenbaum J, Dousa P. Valgus intertrochanteric osteotomy for malunion and nonunion of trochanteric fractures. J Orthop Trauma. 2003; 17(9): 606-12. 10.1097/00005131- 200310000-00002.

9. Kaufer H, Matthews LS, Sonstegard D. Stable fixation of intertrochanteric fractures. J Bone Joint Surg Am. 1974; 56(5): 899907.

10. Wu CC, Shih CH, Chen WJ, Tai CI: Treatment of femoral neck nonunions with a sliding compression screw: comparison with and without subtrochanteric valgus osteotomy. J Trauma. 1999; 46: 312-317. 10.1097/00005373-199902000-00019.

11. Hartford JM, Patel A, Powell J. Intertrochanteric osteotomy using a dynamic hip screw for femoral neck nonunion. J Orthop Trauma. 2005; 19: 329-33.

12. Mueller ME. The intertrochanteric osteotomy and pseudoarthrosis of the femoral neck. 1957. Clin Orthop Relat Res. 1999; (363): $5-8$.

13. Gupta S, Kukreja S, Singh V. Valgus osteotomy and repositioning and fixation with a dynamic hip screw and a $135^{\circ}$ single-angled barrel plate for un-united and neglected femoral neck fractures. J Orthop Surg (Hong Kong). 2014; 22(1): 13-7. doi: $10.1177 / 230949901402200106$ 